\title{
Influence of Magnetic Field on Magnetostructural Transition in
}

\section{$\mathrm{Ni}_{46.4} \mathrm{Mn}_{32.8} \mathrm{Sn}_{20.8}$ Heusler Alloy}

Patricia Lázpita ${ }^{1}$, Volodymyr A. Chernenko ${ }^{1,2, a}$, Jose M. Barandiarán ${ }^{1}$, Iñaki Orue ${ }^{3}$, Jon Gutiérrez ${ }^{1}$, Jorge Feuchtwanger ${ }^{1}$, Jose Alberto Rodriguez-Velamazán ${ }^{4}$

\author{
${ }^{1}$ Universidad del País Vasco, Dept. Electricidad y Electronica, , Sarriena s/n 48940 Leioa, Spain \\ ${ }^{2}$ IKERBASQUE, Basque Foundation for Science, 48011 Bilbao, Spain \\ ${ }^{3}$ SGiker, Vicerrectorado de Inv. UPV/EHU, Sarriena s/n 48940 Leioa, Spain \\ ${ }^{4}$ Institute Laue-Langevin, 6 Rue Jules Horowitz, F-38042-Grenoble Cedex 9, France \\ aladimir.chernenko@gmail.com
}

Keywords: $\mathrm{Ni}_{46.4} \mathrm{Mn}_{32.8} \mathrm{Ga}_{20.8}$ Heusler alloy, magnetostructural transition, martensitic structure

\begin{abstract}
A Mn-enriched $\mathrm{Ni}_{46.4} \mathrm{Mn}_{32.8} \mathrm{Sn}_{20.8}$ Heusler alloy has been prepared by the induction melting and casting method. The samples used were annealed at $900^{\circ} \mathrm{C}$ during $72 \mathrm{~h}$ with a subsequent water quench. The magnetostructural transformation (MST) in the vicinity of $\mathrm{T}_{\mathrm{m}}=230 \mathrm{~K}$ and $\mathrm{T}_{\mathrm{a}}=250 \mathrm{~K}$ has been found, and characterized by $\mathrm{DSC}$, magnetization and resistivity measurements. Changes in the crystal structure due to the MST were observed using neutron diffraction at different temperatures. The crystal structure of martensitic phase shows a good fits to the 4-layered orthorhombic martensite with a Pmma space group. The linear shift of transformation temperatures was found in the entire high magnetic field range up $14 \mathrm{~T}$ to be $\mathrm{dT}_{\mathrm{m}} / \mathrm{dB}_{0}=-1.50 \mathrm{~K} / \mathrm{T}$ and $\mathrm{dT}_{\mathrm{a}} / \mathrm{dB}_{0}=-1.26 \mathrm{~K} / \mathrm{T}$, whereas in the low field range we found an increase of both characteristic temperatures. The entropy changes at the MST were evaluated using the Clausius-Clapeyron relationship. The transformation-induced magnetoresistance has been measured.
\end{abstract}

\section{Introduction}

Ferromagnetic compounds, such as Mn-enriched, non-stoichiometric Ni-Mn-Z Heusler alloys, where $\mathrm{Z}=\mathrm{Ga}, \mathrm{In}, \mathrm{Sn}$, have attracted much interest because they demonstrate unusual magnetoelastic, magnetocaloric and magnetoresistance properties. This is due to the strong coupling of the structural and magnetic degrees of freedom, especially in the vicinity of the martensitic magnetostructural transformation (MST), which they exhibit during cooling from the ferromagnetic cubic phase (see, e.g., [1-3] and references therein). The MST is accompanied by a large drop of the magnetic moment due to the formation of paramagnetic, antiferromagnetic or ferrimagnetic, martensite (such event was mentioned in [4] and experimentally observed in [5]). The magnetic state of the martensite has its origin in the coexistence of competing ferro- and antiferromagnetic (FM-AFM) interactions caused by the different site occupations of $\mathrm{Mn}$ atoms in the L2 $2_{1}$-type ordered lattice [6,7]. These concurrent interactions are encountered in both cubic and martensitic phases, but AFM coupling is stronger in the latter phase due to the change in interatomic distances between neighboring $\mathrm{Mn}$ atoms in the $\mathrm{Z}$ sublattice [8]. In fact the actual magnetic structure of such martensites has never been determined.

The structural, magnetic and magnetocaloric properties have been already studied for the $\mathrm{Ni}_{50} \mathrm{Mn}_{25+\mathrm{x}} \mathrm{Sn}_{25-\mathrm{x}}$ series of materials, focusing on the compound with $\mathrm{x}=14$ [3,6,8-12], while the behavior of Ni-Mn-Sn with deficient content of both $\mathrm{Ni}$ and $\mathrm{Sn}$ in favor of $\mathrm{Mn}$ has so far not received enough consideration. According to the phase diagram 'transformation temperatures versus electron concentration (e/a)' obtained for the aforementioned series [11], the alloy with stoichiometric composition, which corresponds to an $\mathrm{e} / \mathrm{a}=7.75$, does not exhibit MST. On the other hand, the EXAFS studies show that the unequal Ni-Mn bond lengths resulting from excess of Mn 
can trigger the instability of cubic lattice, causing it to transform martensitically [6]. It was interesting to expect this would happen for the $\mathrm{Ni}_{46.4} \mathrm{Mn}_{32.8} \mathrm{Sn}_{20.8}$ alloy with e/a=7.76 selected in the present work to examine its structural and magnetic instabilities under a magnetic field.

\section{Experimental details}

The 140g-ingot of Ni-Mn-Sn alloy was prepared by induction melting and casting into copper mould. The samples were spark-cut and sealed in an evacuated quartz ampoules and annealed at $900^{\circ} \mathrm{C}$ during $72 \mathrm{~h}$, followed by quenching in cold water. The X-ray fluorescent analysis provided an alloy composition of $\mathrm{Ni}_{46.4} \mathrm{Mn}_{32.8} \mathrm{Sn}_{20.8}$ (at.\%), corresponding to an electron-to-atom ratio (e/a) equal to 7.76. Given the large difference between the nuclear scattering lengths of $\mathrm{Mn}$ and $\mathrm{Ni}$, the crystallographic structure at different temperatures could be better studied by neutron diffraction. These experiments were done at the Institut Laue Langevin's (ILL), D1A diffractometer, in Grenoble, France. Diffraction patterns were obtained at seven temperatures between $5 \mathrm{~K}$ and $350 \mathrm{~K}$. The data was collected in the $\theta-2 \theta$ geometry, with a step size of $0.05^{\circ}$ over the range of $20^{\circ}<2 \theta<160^{\circ}$. The neutron wavelength was $0.191 \mathrm{~nm}$.

Magnetization loops and thermomagnetization curves in fields up to $14 \mathrm{~T}$ were obtained using a vibrating sample magnetometer (VSM) from Cryogenic Ltd. Precise measurements of the relative change in electrical resistivity as a function of temperature and magnetic field were carried out by four-probe measurements in a measuring platform from Cryogenic Ltd., for fields up to $14 \mathrm{~T}$. The magnetic field was applied parallel to the measuring electrical current, and the resistance was obtained by averaging the forward and reverse voltage drops at the sample. Accuracy in the absolute resistivity values was about $5 \%$ due to geometrical uncertainty in the samples, while repeatability was better than $0.02 \%$ in different runs of the same sample.

\section{Results and discussion}

Fig. 1(a) shows powder neutron diffraction patterns at different temperatures. Two patterns, 350K and $270 \mathrm{~K}$, correspond to paramagnetic and ferromagnetic cubic phase, respectively, other patterns obviously correspond to the martensitic phase. The occurrence of the martensitic transformation in
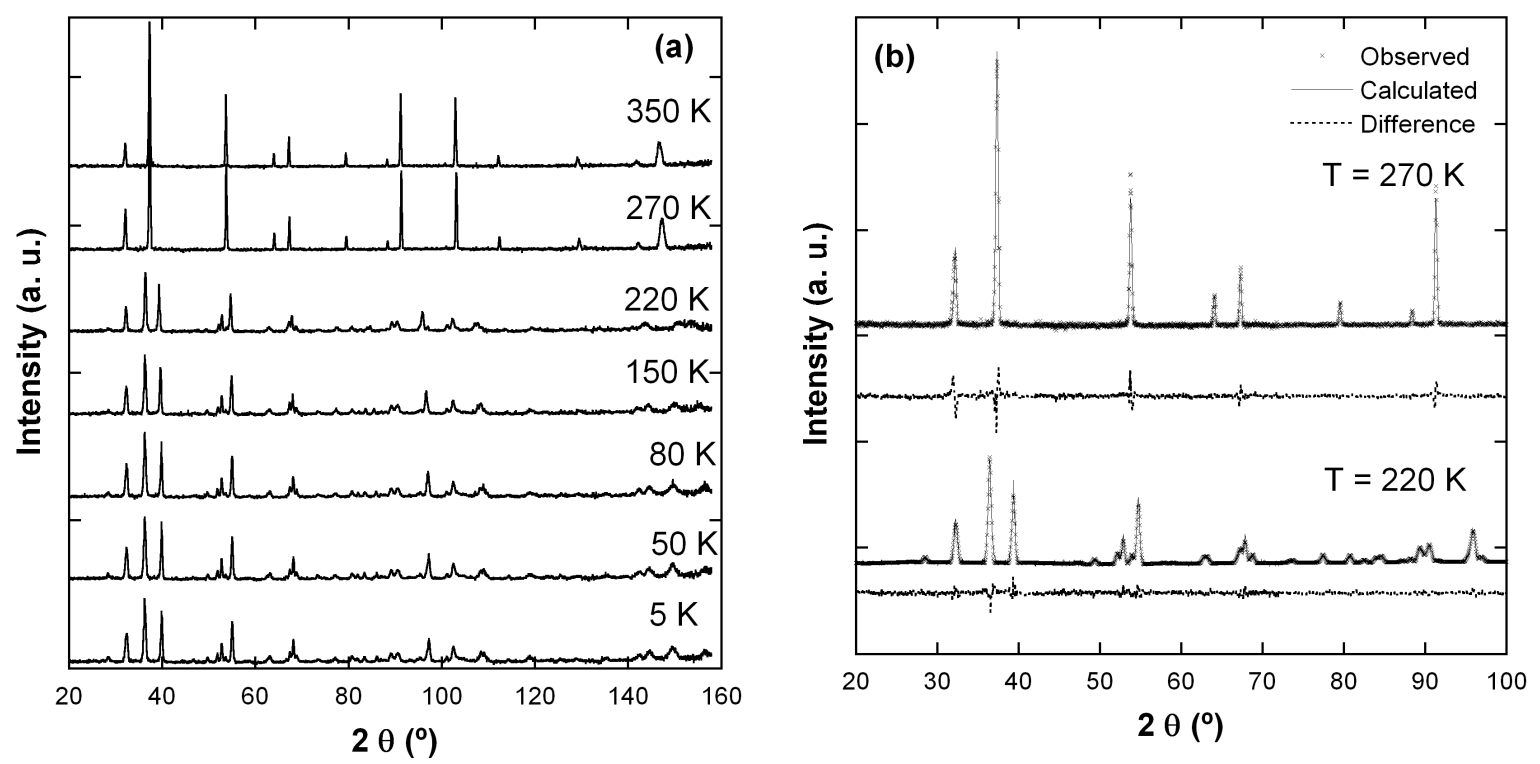

Fig. 1: Neutron diffraction patterns of $\mathrm{Ni}_{46.4} \mathrm{Mn}_{32.8} \mathrm{Sn}_{20.8}$ at different temperatures (a). A comparison between calculated and observed profiles in the cubic Fm-3m L2 $2_{1}$ phase at $270 \mathrm{~K}$ and in the martensitic 4-layered orthorhombic Pmma phase at 220K (b). 
the alloy studied implies an additional lattice instability which is produced by the $\mathrm{Ni}$ for $\mathrm{Mn}$ substitution rather than by the factor of the electron concentration. Note that e/a value almost coincides with this value for the martensitically stable, stoichiometric $\mathrm{Ni}_{2} \mathrm{MnSn}$.

Crystal structure was fitted using the program FullProf, using the space group Pmma, the starting values for the lattice parameters were the ones reported in Refs.[6,9,13]. The calculated and observed diffraction patterns are shown in Fig. 1(b). A good fit, as can be seen in Fig. 1(b), is obtained for both the Fm-3m cubic phase and the Pmma orthorhombic 4-layered martensite.

The lattice parameters from the structure refinement are collected in the Table 1. The neutron diffraction data reveal well-pronounced superstructure reflections, evidencing a high degree of L $2_{1}$ order in studied alloy. The martensitic structure, which is well-described by the set of parameters given in Table 1, is typical for Ni-Mn-Sn alloys [8,9]. The volume thermal expansion coefficient of martensitic phase estimated from the linear dependence in Fig. $2(\mathrm{~b})$ is equal to $1.5 \times 10^{-5} \mathrm{~K}^{-1}$.

Table 1: Structural parameters and transformations characteristics of $\mathrm{Ni}_{46.4} \mathrm{Mn}_{32.8} \mathrm{Sn}_{20.8} . \mathrm{T}_{\mathrm{C}}, \mathrm{T}_{\mathrm{m}}$ and $\mathrm{T}_{\mathrm{a}}$ are the Curie temperature of austenite and forward and reverse martensitic transformation temperatures, respectively.

\begin{tabular}{|c|c|c|c|c|c|c|c|}
\hline \multicolumn{3}{|c|}{ Structure of austenite and martensite } & \multirow{2}{*}{$\begin{array}{c}\mathrm{T}_{\mathrm{C}}, \\
\mathrm{K}\end{array}$} & \multirow{2}{*}{$\begin{array}{c}\mathrm{T}_{\mathrm{m}}, \\
\mathrm{K}\end{array}$} & \multirow{2}{*}{$\begin{array}{l}\mathrm{T}_{\mathrm{a}}, \\
\mathrm{K}\end{array}$} & \multirow{2}{*}{$\begin{array}{c}\mathrm{dT}_{\mathrm{m}} / \mathrm{dB}_{0} \\
\mathrm{~K} / \mathrm{T}\end{array}$} & \multirow{2}{*}{$\begin{array}{c}\mathrm{dT}_{\mathrm{a}} / \mathrm{dB}_{\mathrm{o}} \\
\mathrm{K} / \mathrm{T}\end{array}$} \\
\hline Lattice unit cell & $\begin{array}{l}\text { Space } \\
\text { group }\end{array}$ & Lattice parameters & & & & & \\
\hline Austenite: & Fm-3m & $\mathrm{a}_{\mathrm{c}}=0.5972 \mathrm{~nm}$ & & & & & \\
\hline $\begin{array}{l}\text { Martensite: } \\
\text { 4-layered } \\
\text { orthorhombic }\end{array}$ & Pmma & $\begin{array}{c}\mathrm{a}=0.8579 \mathrm{~nm}, \\
\mathrm{~b}=0.5997 \mathrm{~nm}, \\
\mathrm{c}=0.4342 \mathrm{~nm}(220 \mathrm{~K})\end{array}$ & 318 & 230 & 250 & -1.6 & -1.3 \\
\hline
\end{tabular}

The transformation behavior of $\mathrm{Ni}_{46.4} \mathrm{Mn}_{32.8} \mathrm{Sn}_{20.8}$ alloy can be observed by the temperature dependencies of low-field magnetization and calorimetric signal (Fig. 2(a) and Inset), as well as the dependencies of the lattice parameters and unit cell volume [Fig. 2(b)]. During cooling, the alloy exhibits a ferromagnetic ordering in the cubic phase below the Curie temperature $\mathrm{T}_{\mathrm{C}}$ (second-order transformation) and then a first-order MST at $\mathrm{T}_{\mathrm{m}}$ (Fig.2 and Table 1). Alongside the transformation temperatures, the calorimetric data in the Inset to Fig. 2(a) yield both the temperature hysteresis of
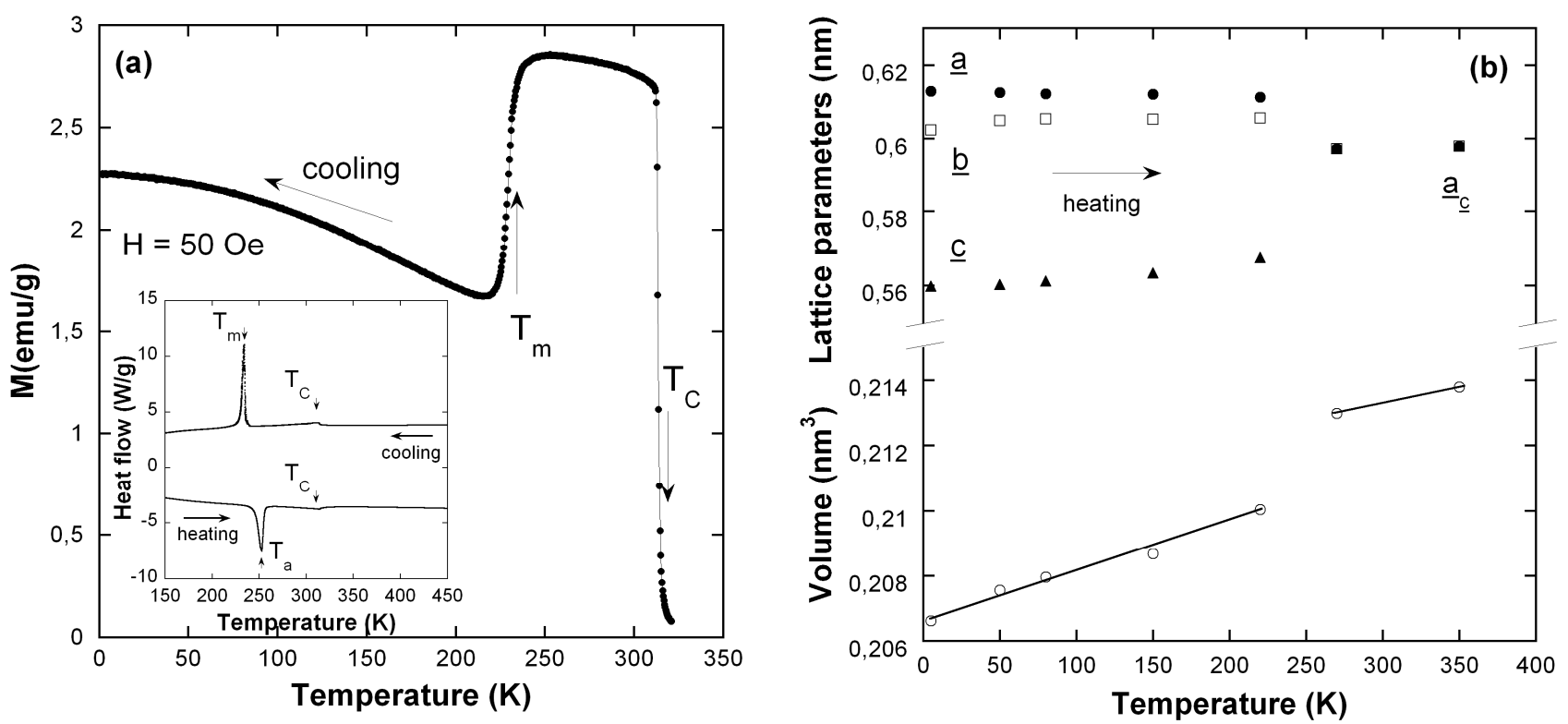

Fig. 2: Transformation behavior of $\mathrm{Ni}_{46.4} \mathrm{Mn}_{32.8} \mathrm{Sn}_{20.8}$ alloy: (a) temperature dependence of low-field magnetization. Inset: DSC profiles; (b) temperature dependence of lattice parameters and cell volume. 
MST of $20 \mathrm{~K}$ and the averaged value of transformation heat of $6.2 \mathrm{~J} / \mathrm{g}$. In agreement with the firstorder nature, the MST is characterized by the abrupt changes of all the physical properties studied, namely magnetization, thermal signal, volume change (Figs.2 and 3) and the resistivity (see below). In particular, the large drop of the magnetic moment during the MST, Fig.3, is crucial for the occurrence of the multifunctional properties of Ni-Mn-Sn alloys $[1,3,5,10,13]$. The widely reported "giant" isothermal properties, such as, the giant magnetostrain or giant magnetoresistance may be explicitly explained by the large, magnetic field-induced, shift of the transformation temperature [14], inherent to Ni-Mn-Z alloy systems with a large excess of Mn [3]. The aforementioned shift is clearly evidenced in Fig.3(a), which shows the evolution of the thermomagnetization curves as a function of magnetic field. In accordance with the signs and values of the saturation magnetization jumps, the magnetic field significantly reduces the MST temperatures. The new finding, is the observation of their increase in the non-saturating fields, so the whole span of $T_{m}$ and $T_{a}$ field dependencies exhibits a maximum, Fig. 3(b). This initial increase can be explained by a slightly 'harder' magnetic behavior of the austenitic phase which is also detectable in the magnetization loops near $\mu_{0} \mathrm{H}=0$ (cf. [14]). The high-field linear parts of curves in Fig. 3(b) yield the slopes shown in Table 1, which are close to those determined for a Ni-Mn-Sn of a different composition [3].

The curves in Fig. 3(a) allows for the extraction of magnetization jumps of the appropriate signs shown in Fig. 4(a). In turn, using the results in Fig.4(a) and derivatives from the Table 1, one can calculate the entropy change accompanying the MST at different values of magnetic field. This is done by substituting the data of Fig 4(a) and shifts from Table 1 into the commonly-known analog of the Clausius-Clapeyron equation [14] resulting in the plots shown in Fig. 4(b). The estimation of $\Delta \mathrm{S}$ value at $\mu_{0} \mathrm{H}=0$ from the data of Fig.4(b) gives the value of transformation heat of about $4.9 \mathrm{~J} / \mathrm{g}$, which is around $20 \%$ less than the value determined from the DSC results. This discrepancy stems from the relative closeness of MST to the Curie temperature.

The phase diagram in Fig. 3(b) points to a reduced actuation capability, stemming from the reversible field-induced MST and accompanying effects in Ni-Mn-Z alloys. This is due to the unfavourable combination of the $T_{m}$ and $T_{a}$ versus $\mu_{0} H$ slopes and the value of transformation temperature hysteresis, resulting in the very high magnetic fields needed to produce a reversible MST in the studied alloy.
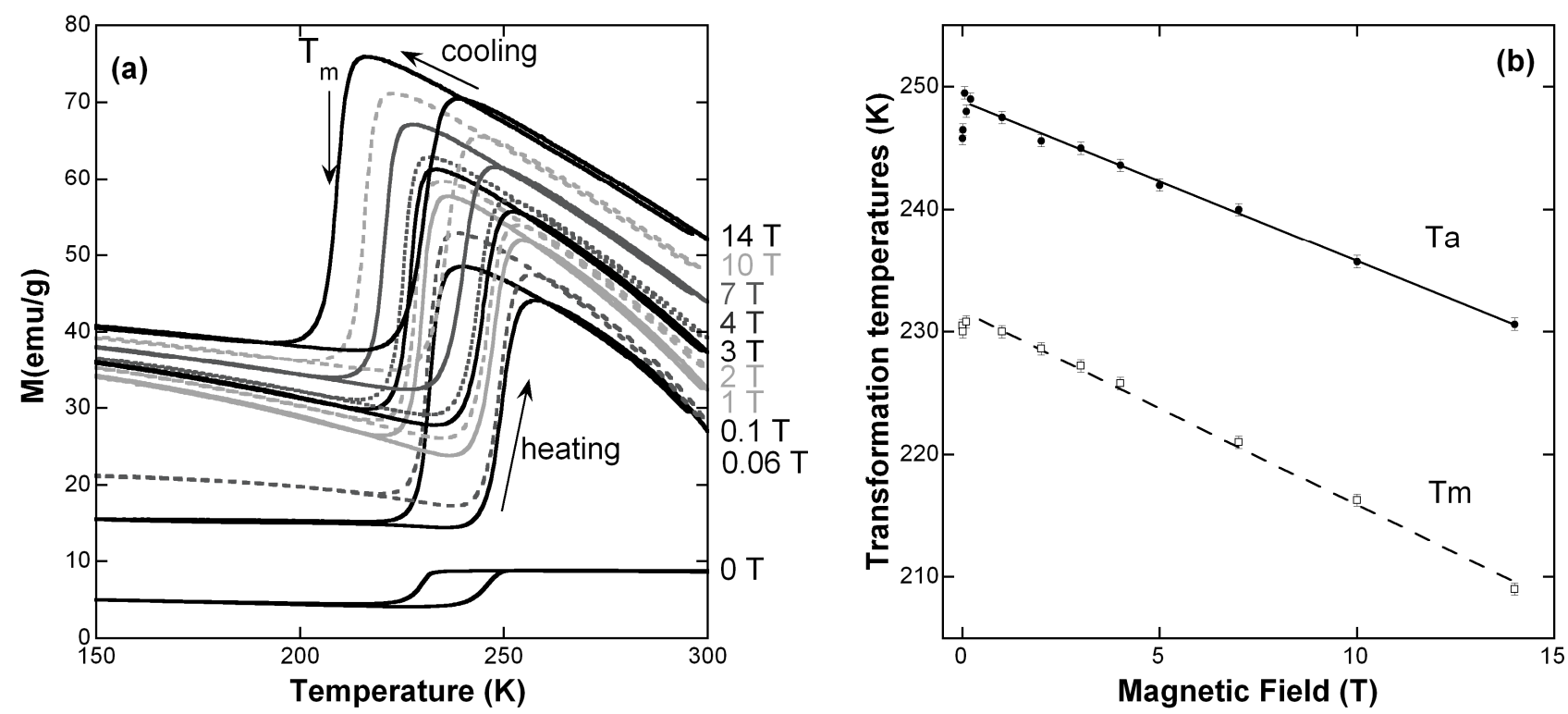

Fig. 3: Cooling-heating thermomagnetization curves measured at the constant magnetic fields (a). Plots of transformation temperatures of MST as a function magnetic field (b). 

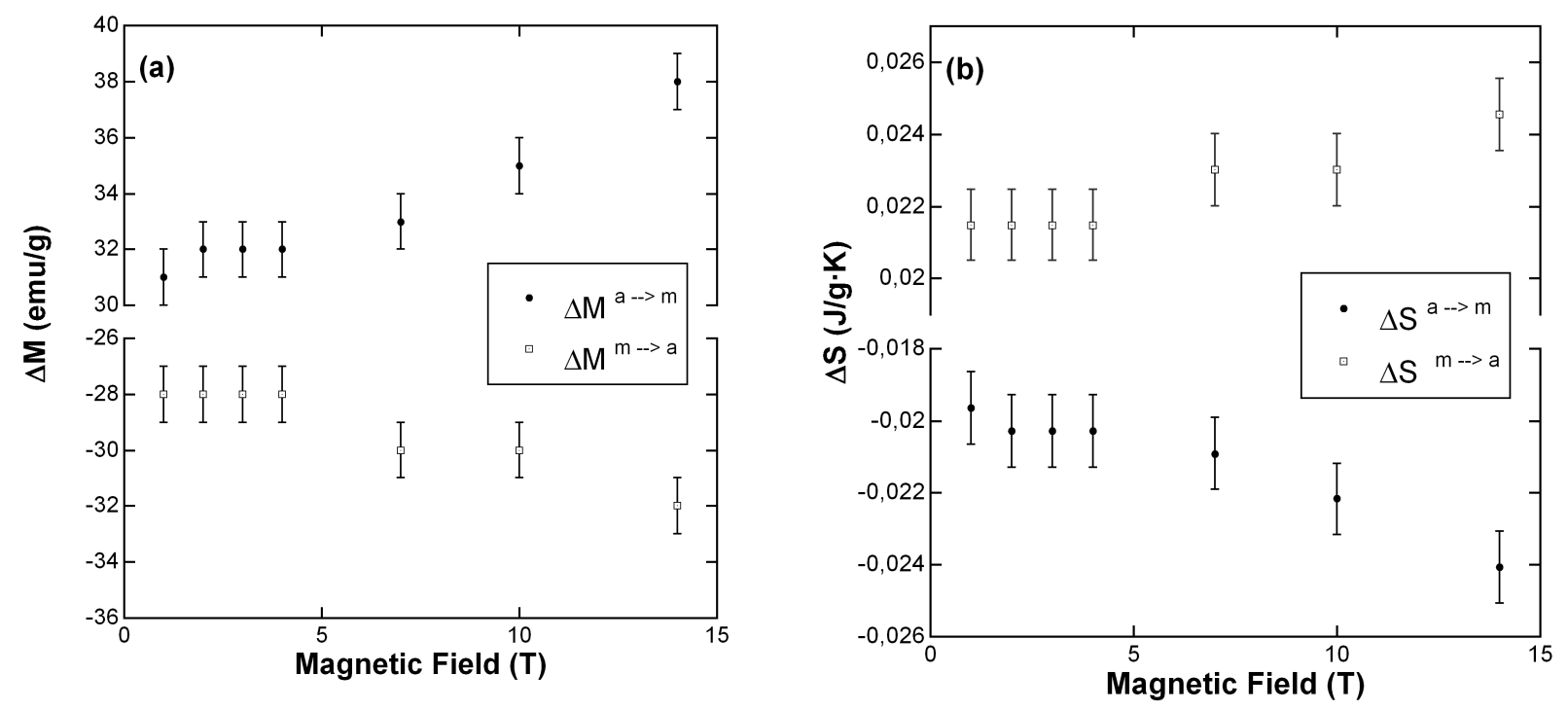

Fig. 4: (a) Magnetic field dependencies of saturated magnetization jumps accompanying the forward and reverse MST as deduced from the plots in Fig. 3(a); (b) field dependencies of entropy change calculated by substituting the data from Fig. 4(a) and shifts from Table 1 into the ClausiusClapeyron equation.

Nevertheless, due to the existence of a temperature interval in which MST proceeds, some volume fraction of the sample can be reversibly transformed under magnetic field at properly selected temperatures. Fig.5 illustrates this situation. Whereas the magnetization loops in the martensitic and austenitic phases show regular field dependencies (Insets to Fig.5), the curve at $234 \mathrm{~K}$ shows an anomalous loop produced by the field-induced MST. The data in Fig.5 and Insets indicate that due to antiferromagnetic component, the magnetization is still not fully saturated at $14 \mathrm{~T}$ and this component is stronger in the low-temperature phase, owing to the increased antiferromagnetic character.

Fig. 6(a) shows resistivity curves at three magnetic fields, which were guides to check the giant magnetoresistance behaviour at selected temperatures in Fig. 6(b). The restricted capability in

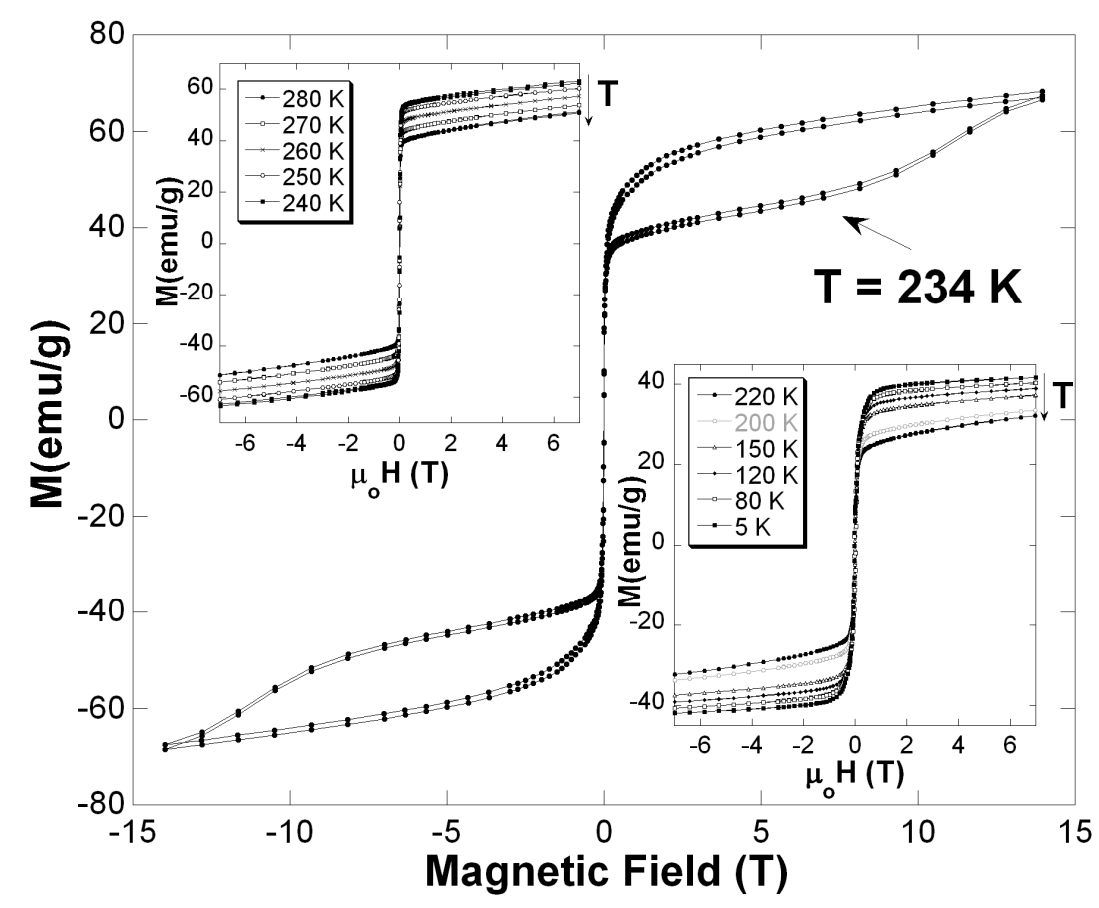

Fig. 5: Magnetization loops measured at different temperatures. 

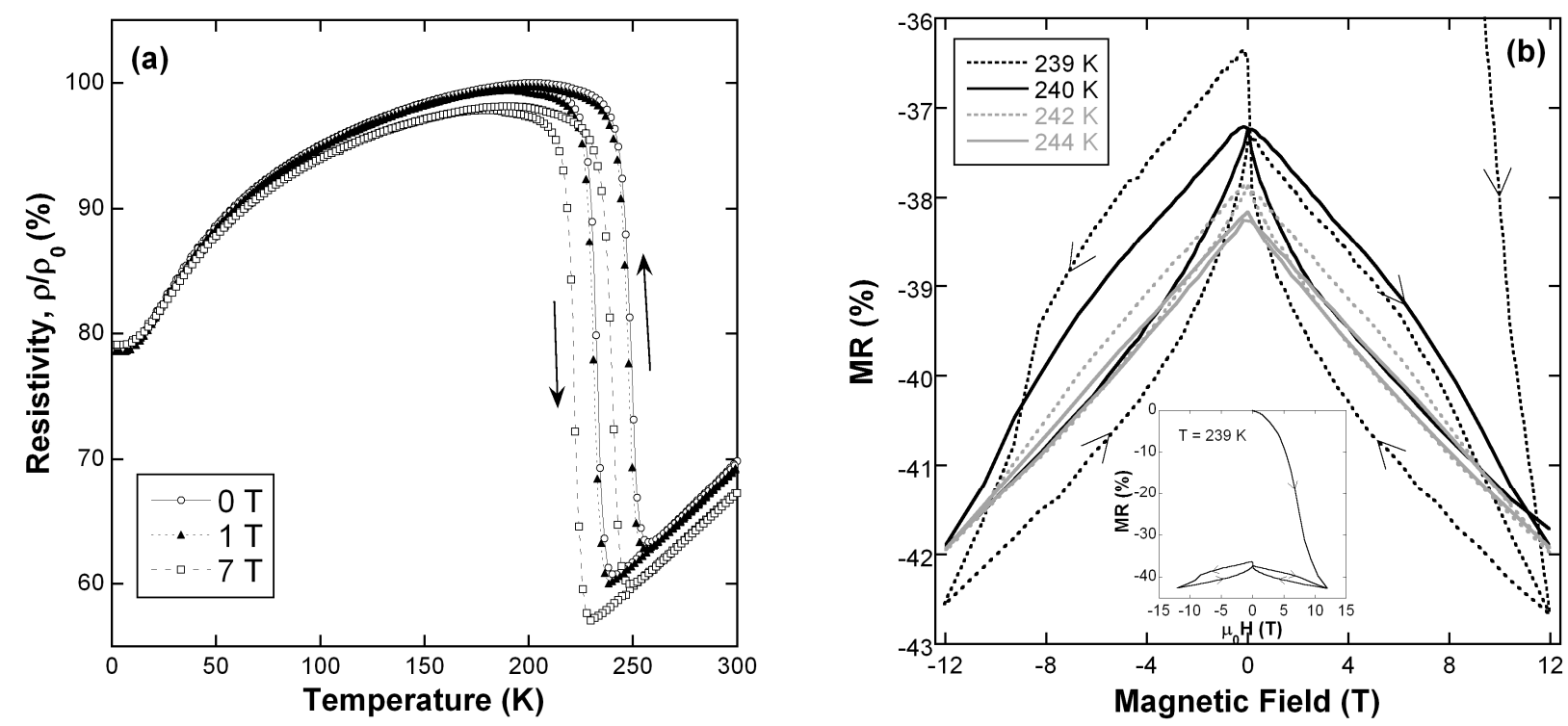

Fig. 6: Temperature dependencies of resistivity at constant magnetic fields (a); magnetoresistance behaviour at the selected temperatures (b).

obtaining large reversible values of actuation is evidenced by the plots in Fig.6(b), where the possibility of getting only about $5 \%$ under $14 \mathrm{~T}$ is experimentally demonstrated. The curve at $239 \mathrm{~K}$ in Fig. 6(b) shows about 45\% drop of resistance under the first field application, due to the complete reverse magnetic field-induced MST, whereas this drop is much smaller during the field unloading owing to the only partially achieved forward MST. In the subsequent field cycling, only partial reverse and forward MSTs take place, giving rise to much lower resistance changes.

\section{Conclusions}

In the present work we found that the $\mathrm{Ni}_{46.4} \mathrm{Mn}_{32.8} \mathrm{Sn}_{20.8}$ alloy with a c/a value close to the one of the stoichiometric $\mathrm{Ni}_{2} \mathrm{MnSn}$ compound exhibits near room temperature a magnetostructural transformation (MST) from a ferromagnetic austenite into a ferromagnetic-less-ordered martensitic phase, as a result of both the structural and magnetic instabilities. These instabilities are introduced by local distortions in the highly atomic-ordered crystal lattice and the component of the antiferromagnetic interactions present in the magnetic subsystem respectively, both effects are due to the excess of Mn occupying "wrong" sites. The crystal structure of martensite is identified as an orthorhombic Pmma cell with a 4-layered lattice modulation. The thermodynamic characteristics of MST are quantitatively determined in the magnetic field range up to 14T. Initial maxima on the transformation temperatures versus magnetic field curves were found. The actuation ability due to the magnetic field-induced MST has been verified on the magnetoresistance measurements.

\section{Acknowledgements}

The financial support from the Department of Education, Basque Government (Project No. IT-34707) and the Spanish Ministry of Education and Science (Project No. MAT2008-06542-C04-02) is acknowledged. J.F. is grateful to the EC Marie Curie IIF Initiative under Grant No. FP6. Authors are grateful to Prof. A. Perekos for the help in the preparation and chemical analysis of the alloy.

\section{References}

[1] Advances in Shape Memory Materials. V.A. Chernenko (Ed.), Mater. Sci. Forum, 583, TTP, Switzerland, 2008, pp.1-302. 
[2] O. Soderberg, Y. Ge, A. Sozinov, S.-P. Hannula and V.K. Lindroos, in: J. Buschow (Ed.), Handbook of Magnetic Materials, 16, Elsevier Science, Amsterdam, 2006, pp. 1-39.

[3] A. Planes, Ll. Mañosa and M. Acet: J. Phys.: Condens. Matter Vol. 21 (2009), p. 233201

[4] V.A. Chernenko, V.A.L'vov, S.P.Zagorodnyuk and T. Takagi: Phys. Rev. B Vol.67 (2003), p. 064407

[5] R. Kainuma, Y. Imano, W. Ito, Y. Sutou, H. Morito, S. Okamoto, O. Kitakami, K. Oikawa and A. Fujita: Nature Vol. 439 (2006), p.957

[6] P. A. Bhobe, K. R. Priolkar, and P. R Sarode: J. Phys.: Condens. Matter Vol.20 (2008), p. 015219

[7] M. L. Richard, J. Feuchtwanger, S. M. Allen, R. C. O’Handley, P. Lázpita, J. M. Barandiarán, J. Gutiérrez, B. Ouladdiaf, C. Mondelli, T. Lograsso and D. Schlagel: Phil. Mag. Vol. 87 (2007), p. 3437

[8] Y. Sutou, Y. Imano, N. Koeda, T. Omori, R. Kainmura, K. Ishida and K. Oikawa: Appl. Phys. Lett. Vol. 85 (2004), p. 4358

[9] P. J. Brown, A. P.Gandy, K. Ishida, R. Kainuma, T. Kanomata, K.-U. Neumann, K. Oikawa, B. Ouladdiaf, and K. R. A. Ziebeck: J. Phys.: Condens. Matter Vol.18 (2006), p.2249

[10]K. Koyama, K.Watanabe, T.Kanomata, R. Kainuma, K. Oikawa and K. Ishida: Appl. Phys. Lett. Vol.88 (2006), p.132505

[11]X. Moya, Ll. Manosa, A. Planes, T. Krenke, M. Acet and E.F. Wassermann: Mater. Sci. Eng. A Vol. 438-440 (2006), p. 911

[12]X. Moya, L1. Manosa, A. Planes, T. Krenke, E. Duman, M. Acet and E.F. Wassermann: JMMM, Vol. 316 (2007), p. e572

[13]B. Hernando, J. L. Sánchez Llamazares, J. D. Santos, Ll. Escoda, J. J. Suñol, R. Varga, D. Baldomir and D. Serantes: Appl. Phys. Lett. Vol. 92, (2008), p.042504

[14]J. M. Barandiarán, V. A. Chernenko, P. Lázpita, J. Gutiérrez, and J. Feuchtwanger: Phys. Rev. B Vol. 80 (2009), p.104404 